\title{
Betaine chemistry, roles, and potential use in liver disease
}

\author{
Christopher R Day and Stephen A Kempson \\ Laboratory of Receptor Biology and Gene Expression, Center for Cancer Research, \\ National Cancer Institute, NIH, Bethesda, MD 20892, USA \\ and \\ Department of Cellular \& Integrative Physiology, Indiana University School of Medicine, \\ Indianapolis, IN 46202-5120, USA
}

Running header: Betaine and liver disease

Correspondence to: Dr SA Kempson, MS 306, 635 Barnhill Dr, Indianapolis, IN 46202-5120, USA

Tel: 317-274-7772. Email: skempson@iupui.edu

\begin{abstract}
Abbreviations
ALD, alcoholic liver disease. AMPK, AMP-activated protein kinase. BGT1, $\mathrm{Na}^{+} / \mathrm{Cl}^{-}-$ dependent betaine-GABA transporter. BHMT, betaine-homocysteine methyltransferase. DMG, dimethylglycine. NAFLD, non-alcoholic fatty liver disease. NASH, nonalcoholic steatohepatitis. OCTN2, organic cation/carnitine transporter. SAH, Sadenosylhomocysteine. SAM, S-adenosylmethionine. SIT1, $\mathrm{Na}^{+}$-dependent imino acid transporter. SNAT, $\mathrm{Na}^{+}$-dependent neutral amino acid transporter.
\end{abstract}

This is the author's manuscript of the article published in final edited form as: Biophysica Acta (BBA) - General Subjects, 1860(6), 1098-1106. http://doi.org/10.1016/j.bbagen.2016.02.001 
BBAGEN-15-673 R2

\section{Abstract}

Background. Betaine is the trimethyl derivative of glycine and is normally present in human plasma due to dietary intake and endogenous synthesis in liver and kidney. Betaine is utilized in the kidney primarily as an osmoprotectant whereas in the liver its primary role is in metabolism as a methyl group donor. In both organs a specific betaine transporter mediates cellular uptake of betaine from plasma. The abundance of both betaine and the betaine transporter in liver greatly exceeds that of other organs.

Scope of review. The remarkable contributions of betaine to normal human and animal health are summarized together with a discussion of the mechanisms and potential beneficial effects of dietary betaine supplements on liver disease.

Major conclusions. A significant amount of data from animal models of liver disease indicates that administration of betaine can halt and even reverse progression of the disruption of liver function. Betaine is well-tolerated, inexpensive, effective over a wide range of doses, and is already used in livestock feeding practices.

General significance. The accumulated data indicate that carefully controlled additional investigations in humans are merited. The focus should be on the long-term use of betaine in large patient populations with liver diseases characterized by development of fatty liver, especially non-alcoholic fatty liver disease and alcoholic liver disease.

Key words: alcohol, hepatocyte, non-alcoholic fatty liver, betaine/GABA transporter, methionine cycle. 


\section{Introduction.}

Betaine is an important human nutrient and human blood plasma typically contains $20-70 \mu \mathrm{mol} /$ liter which is not protein-bound. It is present in almost all tissues but the highest concentrations are in liver, kidney, and testes [1]. Some foods, such as whole grains, spinach, shrimp and beets, are rich in betaine and dietary intake is an important source. In the USA, the average dietary betaine intake is about 100-300 $\mathrm{mg}$ /day and rarely exceeds 400-500 mg. In addition, there is endogenous synthesis of betaine from choline, an essential nutrient, in the liver and also in the kidney. Excellent comprehensive summaries of some important biological roles of betaine have appeared in recent years [2-5]. One purpose here is to highlight recently emerging data on the therapeutic use of betaine and the possible mechanisms by which it can attenuate liver injury, especially in rodent models.

\section{Chemistry.}

Betaine is the common term, and the one used throughout this review, that refers to a methyl derivative of glycine. The term glycine betaine is used increasingly to distinguish it from other betaine derivatives such as proline betaine and alanine betaine. Betaine (CAS \# 107-43-7) is known also as trimethylglycine or N,N,Ntrimethylammonioacetate (IUPAC name). The pharmaceutical form of anhydrous betaine is marketed as Cystadane ${ }^{\circledR}$. It was first discovered as a natural byproduct when sugar beets (Beta vulgaris) were processed for extraction of sucrose, and eventually was found to have a widespread distribution in many microorganisms, marine invertebrates, plants and animals. Beet is still the main source of commercially available betaine, and some physical and chemical properties of betaine are listed in Table 1. Over the physiological 
$\mathrm{pH}$ range it is a zwitterion with a positively charged tri-methylammonium group and a negatively charged carboxyl group (Fig 1) and thus requires no counterions for electroneutrality in the cytoplasm. Betaine is stable, non-toxic and highly soluble in water. Strong acids convert anhydrous betaine to various salts. For example, hydrochloric acid produces betaine hydrochloride:

$$
\left(\mathrm{CH}_{3}\right)_{3} \mathrm{~N}^{+} \mathrm{CH}_{2} \mathrm{CO}_{2}^{-}+\mathrm{HCl} \rightarrow\left[\left(\mathrm{CH}_{3}\right)_{3} \mathrm{~N}^{+} \mathrm{CH}_{2} \mathrm{CO}_{2} \mathrm{H}\right] \mathrm{Cl}^{-}
$$

The hydrochloride form (CAS \# 590-46-5) is used primarily as a source of hydrochloric acid for people with hypochlorhydria (low stomach acid). In the alkaline environment of the small intestine betaine hydrochloride is converted to betaine.

When betaine is released into the environment by excretion or death, especially in hypersaline environments, a variety of aerobic and anaerobic microorganisms degrade betaine. The degradation products include trimethylamine $\left(\mathrm{CH}_{3}\right)_{3} \mathrm{~N}$ and methylamine $\mathrm{CH}_{3} \mathrm{NH}_{2}$ from a number of different pathways [6-7]. Synthesis of betaine and subsequent demethylation in liver plays a vital role in one-carbon metabolism (Fig. 2).

Betaine's unique actions as an osmolyte and chemical chaperone are due to its underlying chemistry. Osmolytes are small organic compounds that are accumulated by cells during stressful external conditions [8]. Organic osmolytes are compatible solutes because their interactions with macromolecules are not detrimental and they can be upand down-regulated without interfering with cellular functions. In contrast, inorganic ions such as $\mathrm{Na}^{+}$and $\mathrm{Cl}^{-}$can destabilize proteins and nucleic acids when present at high concentrations [9]. Additional interest in this group of compounds is due to their ability as chaperones to modify the structure and stability of macromolecules such as proteins and DNA [10]. This effect of betaine and similar osmolytes involves universal water- 
solute-macromolecule interactions. Whereas destabilizers like ions and urea bind to proteins and cause them to unfold, betaine has the opposite effect. It does not bind and is excluded from protein's immediate hydration layer causing the protein to fold more compactly, a so-called 'osmophobic' effect [8]. In brief, betaine becomes distributed nonuniformly due to exclusion from proteins and this produces a thermodynamic force that drives proteins into a smaller volume in order to reduce the amount of excluded water. This stabilizes the proteins' native structure. By focusing the osmophobic force on the denatured state, the native state is left free to function relatively unfettered by the presence of osmolyte [11]. This is supported by recent studies on the mechanism of protein stabilization by betaine [10] that showed a link between the influence of betaine (and other osmolytes) on water structure and the ability to stabilize protein structure. In aqueous solution betaine is easily and tightly hydrated by strong hydrogen bonding with water molecules that extends from the carboxylate to the methyl groups (Fig. 1). There is easy exchange of water molecules between the hydration sphere of betaine and the bulk water, and the statistical average number of water molecules remaining constantly around the betaine molecule is very low [12]. Both values suggest that one water molecule, when attracted by a betaine molecule, is very rapidly substituted by another water molecule meaning that betaine does not immobilize water molecules. Water localization increases as betaine concentration increases and up to 12 water molecules can reside within the hydration shell of each betaine molecule with strong intramolecular forces acting between them and providing complex stability. Thus betaine enhances the surrounding water structure that excludes it from the immediate hydration layer of peptide backbones.

\subsection{An intriguing question.}


If betaine is excluded from protein surfaces how does it bind to transport proteins or enzymes ? The principal problem for binding is the very bulky quaternary amine group which has a positive charge distributed over a large volume resulting in a much smaller surface potential compared with a metal cation. It cannot compete with small water molecules (with larger surface potentials) for binding sites on the surface of the transporter or enzyme protein. Clues were obtained from studies of betaine binding to a highly specific and high affinity betaine transport system (ProU) of E.coli. The binding protein of the ProU transport system solves this problem by forming a cavity with evenly distributed negative surface potential that is just large enough to accommodate the quaternary amine group of betaine. The positive charge of the quaternary amine forms noncovalent cation- $\pi$ interactions with electron-rich $\pi$ bonds of the aromatic rings of three tryptophan residues in the cavity, termed an aromatic box, of the transport protein [13]. Such a specific requirement for binding a quaternary amine group likely avoids a considerable amount of non-specific binding to the betaine binding site of the transporter or enzyme protein. The high affinity for betaine overcomes the osmophobic forces that restrict binding.

\section{Roles of betaine in mammals.}

Betaine has several vital roles in mammalian physiology and more are being uncovered (Fig. 3). Both the kidneys and liver, and perhaps other organs, contain cells that express specific transport systems to mediate uptake and accumulation of betaine from the blood supply [2-4]. The primary role in the kidney is osmoprotection in the cells in the inner medulla where the osmolarity is always high, but changing. Cellular adaptation to these conditions is a complex process $[14,15]$ that includes accumulation of 
betaine from the blood via the $\mathrm{Na}^{+}$- and $\mathrm{Cl}^{-}$-dependent betaine-GABA transporter (BGT1) transporter in the basal plasma membrane. Intracellular betaine, together with other organic molecules (osmolytes), balances the high extracellular osmolarity and maintains normal cell volume. As discussed earlier, betaine is an example of a nonperturbing osmolyte because it can be accumulated to high concentrations ( $\mathrm{mmol} /$ liter) without deleterious effects on the cells. Also, through its chaperone effect, intracellular proteins are protected from the denaturing effects of urea and $\mathrm{NaCl}$ which are present at high concentrations in the kidney medulla $[16,17]$.

The betaine content of liver greatly exceeds that of other organs, except the kidney [1], and the abundance of the BGT1 transport protein is highest in liver compared to other organs [18]. Betaine in liver functions primarily as a methyl donor in one-carbon metabolism and there has been renewed interest in the therapeutic use of betaine for reducing liver injury. One-carbon metabolism is a metabolic network of interdependent biosynthetic pathways compartmentalized in the cytoplasm, mitochondria, and nucleus. For example, one-carbon metabolism in the nucleus plays a critical role in DNA replication and repair and in DNA methylation, a regulatory epigenetic process [19]. A simple abnormality in the methylation pathway, compounded with further assaults from environmental and infectious agents can lead to a wide range of conditions including, for example, cardiovascular disease, neurotransmitter imbalances, cancer, diabetes, abnormal immune function, and chronic inflammation. In the cytoplasm betaine is required for the remethylation of homocysteine to methionine [20, 21], a precursor to Sadenosylmethionine (SAM), the universal methyl donor in the body. SAM donates its labile methyl group to more than 80 biological methylation reactions. Cells generate 
SAM via the one-carbon pathway for transfer of a methyl group (Fig. 4). The methyl groups are provided by two donors, betaine and 5-methyltetrahydrofolate.

Methyl groups are transferred from betaine to homocysteine in a reaction catalyzed by betaine-homocysteine methyltransferase (BHMT, Fig.4), the methionine cycle. It is notable that in both mouse and humans BHMT is most abundant in liver but also expressed in kidney $[18,22]$. BHMT is a zinc metalloprotein, and catalysis follows an ordered bi-bi mechanism where homocysteine is the first substrate to bind to BHMT and dimethylglycine is the first product to leave [23]. Dimethylglycine is a strong noncompetitive inhibitor of BHMT, and betaine will not bind in the absence of homocysteine [24]. Homocysteine binding is facilitated by the presence of $\mathrm{K}^{+}$ions which reduces the Michaelis constant $\left(\mathrm{K}_{\mathrm{m}}\right)$ for homocysteine but not betaine [25]. BHMT-2 has $80 \%$ identity to BHMT but cannot use betaine as the methyl donor, and shows almost no inhibition by dimethyl glycine [26, 27]. Methyl groups are transferred from 5methyltetrahydrofolate to homocysteine by methionine synthase (present in all tissues) in the folate cycle (Fig. 4), using methylcobalamin as a cofactor [2, 21]. Both enzymes contribute equally to remethylation of homocysteine in liver [2].

The major external source of methyl groups comes from the dietary supply of methionine, although dietary choline (metabolic precursor of betaine) and folate also are important [28]. Depletion of dietary folate alone is sufficient to diminish the methyl pool [29] and alcohol, a folate antagonist, also can deplete the methyl pool. Excess consumption of alcohol impairs folate absorption by inhibiting expression of the folate transporter, thus decreasing the hepatic uptake and renal conservation of circulating folate. Alcohol consumption by rodents also produced significant decreases in liver 
BBAGEN-15-673 R2

content of both SAM and betaine [30, 31].

Therapeutic applications of betaine have been used in both humans and animals. In humans betaine ( $6 \mathrm{~g} /$ day or more) has been effective in treating severe hyperhomocysteinemia, a risk factor for vascular complications and neurodegenerative diseases $[3,32]$ that arises due to the hereditary genetic disorder homocysteinuria. Betaine supplements in patients with mild hyperhomocysteinemia led to a rapid reduction of plasma homocysteine levels that was maintained over the long term [2]. Use of betaine in animal husbandry is well-established. For many years it has been included in livestock feeds and is a safe method [33] to increase the lean muscle mass and decrease the fat of meat. This application consumes a major part of the world's betaine production. Its effectiveness is best documented in pigs, but similar responses are known in poultry and in lambs $[3,34]$. How betaine affects body fat is not clear, but it has caught the attention of the bodybuilding and athletic performance industry. There are suggestions that betaine supplements can reduce fatigue and aid performance and recovery $[2,3]$, but research in human subjects has shown no effect on body weight or body composition [35].

More recently betaine was detected in mouse oviducts where it appears to increase after fertilization. It may act as an osmolyte in the developing embryo because preimplantation embryos accumulate betaine via the $\mathrm{Na}^{+}$-dependent imino acid transporter (SIT1). Further, the detection of endogenous betaine synthesis, high betaine concentrations, and BHMT activity in the mouse blastocyst suggests the importance of betaine in methylation reactions early in development. This is supported by observations 
that inhibition of BHMT during embryogenesis resulted in developmental defects [36, 37].

\section{Betaine in non-mammalian systems}

Betaine has been shown to have additional actions in non-mammalian systems, such as nematode worms, but the possible relevance to mammalian physiology has not been determined $[38,39]$. Betaine also has found use as a cryoprotectant for frozen storage of microorganisms [40].

Inclusion of betaine in feed used by salmon farms may help young fish raised in freshwater to maintain osmotic balance and grow faster when transferred abruptly to seawater $[41,42]$. However, the data are few and not very convincing.

As a zwitterion, and a small polar molecule prone to hydrogen bonding, betaine strongly interacts with water and other similar molecules, giving a 'silkier' feel to solutions, which explains its use in personal care products such as moisturizers and toothpaste [3].

The polymerase chain reaction (PCR) process, and other DNA polymerase-based assays, such as DNA sequencing, can be enhanced by betaine. The amplification of contorted GC-rich regions and the product yield are improved in the presence of betaine. The mechanism is unknown but it may be due in part to facilitating strand separation and prevention of secondary structures in the DNA molecules. The overall effect is to equalize the contribution of GC-base pairs (strong) and AT- base pairs (weak) to the stability of the DNA duplex $[43,44]$. The effect of betaine on the fidelity of the polymerase is another unknown, but it does not perturb the polymerase-DNA interaction or initial annealing of primer. In addition, the chemical chaperone action of betaine may 
improve the resistance of DNA polymerase to denaturation. Experiments indicate betaine is best used at a final concentration of $0.8-1.6 \mathrm{M}$ although optimal conditions may need to be determined for specific amplification reactions [44, 45].

\section{Betaine transport in kidney and liver}

Plasma betaine is filtered at the glomerulus of the kidney and is conserved efficiently by reabsorption along the nephron. The low fractional excretion of betaine indicates that filtered betaine is nearly completely reabsorbed from the tubular lumen in the proximal tubule and long loops of Henle [46]. The transport system that reabsorbs betaine in the proximal tubule resides in the luminal plasma membrane and may be the SIT1 transporter [47]. This is distinct from the BGT1 transporter in the medullary segments of the nephron that transports betaine from the blood supply and into the cells where it can accumulate, as discussed in section 3. Notably, the synthesis and expression of BGT1 is induced by osmotic stress and the mechanisms involved in these processes have been discussed already in several excellent reviews [14, 48-50]. The response to osmotic stress is relatively slow, at least in cultured cells, compared to acute downregulation by adenosine and upregulation by nitric oxide [51]. Some requirements for accurate targeting of BGT1 to the basolateral plasma membrane during osmotic stress have been reported by ourselves and others [52-56]. However while the structural and functional relationships of the bacterial betaine transporter BetP have been identified at the molecular level [57], similar analysis of the BGT1 protein remains superficial.

Recent data has shown that BGT1 is much more abundant in liver compared to kidney [18]. We confirmed the presence of BGT1 in hepatocyte plasma membranes and showed it is functional under iso-osmotic conditions. These initial studies also raised the 
possibility that some betaine uptake in hepatocytes could utilize the ubiquitous transport systems for amino acids (SNAT) and carnitine (OCTN2). The multiple pathways for betaine accumulation likely reinforces the importance of betaine in normal liver function. However, the relative contributions of betaine uptake and betaine synthesis in maintaining liver betaine content is an unanswered question [31]. In developing rats the dietary betaine may be more important than synthesis [58].

\section{Liver diseases.}

Many different pathogenetic agents and processes can affect the liver and cause diseases. Viruses like hepatitis A, B and C cause some of them. Others can be the result of drugs, toxins, excess alcohol consumption, and hereditary diseases. Those arising from acute damage to the functional cells of the liver (principally hepatocytes) without destroying regenerative capacity are generally reversible. Other consequences of liver disease are irreversible such as cirrhosis (scarring) where the liver becomes hard, shrunken and nodular and the amount of functioning tissue is decreased leading to impaired function. Despite having many different causes, a common feature of many liver diseases is fat accumulation in liver cells. Fatty liver (hepatic steatosis) occurs worldwide in the obese and in those with excessive alcohol intake. Here we focus on a significant body of evidence suggesting a novel therapeutic opportunity for the use of betaine on non-alcoholic fatty liver disease (NAFLD) caused by a combination of hereditary and environmental risk factors, and alcoholic liver disease (ALD) caused by alcohol abuse.

NAFLD is a common chronic liver disease worldwide with a prevalence that increases with increasing body weight. It is the most common cause of liver disease in 
children and adolescents [59]. NAFLD includes a spectrum of abnormalities ranging from triglyceride accumulation in hepatocytes (often symptom-free) to non-alcoholic steatohepatitis (NASH). The latter involves infiltration of inflammatory cells and necrosis due to fat accumulation and can progress to cirrhosis of healthy tissue and liver failure. It is an increasing cause of liver transplantation. The mechanisms involved in NAFLD are not completely understood but one hypothesis for hepatocyte injury is the "two-hit" proposal [60]. The first hit is insulin resistance which leads to hepatic fat accumulation, characterized by macro- and micro-vesicular lipid droplets. The second hit involves mitochondrial dysfunction, lipid peroxidation and proinflammatory cytokines, leading to more serious damage and hepatocyte injury [61, 62]. NASH is of particular concern because it has significant potential for development of cirrhosis and its complications. Management is often directed at aggressive reduction of the risk factors, namely obesity and insulin resistance [63]. Insulin resistance, reflected as hyperinsulinemia, is positively correlated with elevated plasma homocysteine in NASH patients [64, 65]. In addition, there is decreased mitochondrial function and altered structure in NASH [66, 67].

ALD occurs after years of heavy alcohol consumption and is associated with fatty liver and progression to alcoholic hepatitis, cirrhosis and liver cancer. It is one of the top ten causes of death in developed countries. Symptoms vary with the severity of the injury and, surprisingly, not all heavy drinkers will develop clinically important ALD [68]. Alcohol consumption also may aggravate liver injury caused by non-alcoholic liver diseases such as chronic hepatitis C. Mild alcoholic hepatitis can be reversed by abstinence. Cirrhosis cannot be reversed but abstinence may prevent further injury. Cirrhosis is a major risk factor for development of hepatocellular carcinoma and it has 
BBAGEN-15-673 R2

been estimated that $1 \%$ of the population worldwide has detectable cirrhosis. The only curative option for excessive cirrhosis is a liver transplant but some pharmacological strategies for halting the progression of cirrhosis are being studied $[69,70]$.

\section{Use of betaine in liver injury due to NAFLD and ALD.}

The use and effectiveness of betaine as a methyl donor supplement in both nonalcoholic and alcoholic liver injury has been investigated. Exogenous betaine is inexpensive, well-tolerated, and has been used to decrease fat content of livestock carcasses and for treatment of hyperhomocysteinemia in humans $[4,34,71]$. It is notable that deletion of BHMT in mice, thus preventing removal of methyl groups from endogenous betaine, was associated with increases in fatty liver and hepatocellular carcinoma [22]. Fat accumulation in the liver may play a critical role not only in disease initiation, but also in the progression to NASH and cirrhosis. It has been proposed that prevention of fat accumulation in the liver may be an effective therapy for multiple stages of NAFLD [72]. Mice fed a moderately high fat diet for several weeks develop NAFLD [62]. However, providing dietary supplements of betaine at the same time increased SAM and prevented betaine deficiency, insulin resistance and steatosis in liver. Furthermore, betaine administered after development of NAFLD reversed both insulin resistance and steatosis. These effects were attributed to normalization of downstream pathways involved in insulin signaling, gluconeogenesis and glycogen synthesis [73]. A similar study in mice reported that betaine restored adipose tissue function and sensitivity to insulin and suggested these actions may be due in part to relief of endoplasmic reticulum (ER) stress [74]. Attenuation of hepatic steatosis in mice by dietary betaine also was found to be associated with increased activation of hepatic AMP-activated protein kinase 
(AMPK) [72], and both findings were confirmed in rats [75]. AMPK has been implicated in control of hepatic glucose and lipid homeostasis via multiple effects on genes and short-term regulation of specific enzymes. It has been suggested that alleviation of hepatic steatosis by betaine administration may be due to enhanced fatty acid oxidation and lipid export [75]. The mechanism may involve the down-regulation of genes involved in de novo synthesis and up-regulation of genes involved in fatty acid oxidation, based on a study of the action of betaine on genomic methylation. The dysregulation of expression of several relevant genes in NAFLD mice was corrected by betaine feeding [67].

In summary, betaine action in NAFLD in rodents appears to involve multiple metabolic pathways. The critical step may be to regulate gene expression involved in fatty acid and lipid metabolism and thereby ameliorate development of hepatic steatosis. In turn, this may protect mitochondria from lipotoxicity due to failure of fatty acid oxidation [67] and relieve ER stress. Some of these effects may be due to indirect actions of betaine. For example, betaine increases expression in liver of fibroblast growth factor 21 which is a metabolic regulator [4].

Although the underlying causes of NAFLD and ALD are clearly different, there are overlapping similarities in the histology, the disturbances in hepatic metabolic pathways, and some factors that appear to drive these changes. These are listed in Table 2 and include insulin resistance, dysregulation of lipid metabolism, cytokines that mediate inflammation, and fibrosis and necrosis of hepatocytes. The role of the proinflammatory cytokine TNF- $\alpha$ in mediating early stages of fatty liver and also the progression to liver injury has been determined from studies of both NASH and ALD [68]. 
Alcohol is thought to cause fatty liver by producing a decrease in fatty acid oxidation, and by stimulating lipogenesis. These effects may be due in part to inhibition of AMPK which controls fatty acid metabolism [76- 78]. Inhibition of AMPK by ethanol has been reported in both mice and in rat hepatoma cell lines [79]. The importance of this effect of ethanol was emphasized by the observation that treatment of ethanol-fed rats with a selective activator of AMPK attenuated the development of fatty liver [80]. Similarly, expression of a constitutively active form of AMPK in rat hepatoma cells also blocked some of the effects of ethanol [79]. Similar to its action in NAFLD, betaine has been reported to be protective of liver injury in animal models of ALD [81-83]. The enzyme methionine synthase, a vital enzyme for remethylation of homocysteine via the folate cycle, is inhibited by ethanol consumption $[84,85]$ and this may account for an increase in homocysteine in plasma. Under these conditions, dietary supplements of betaine likely provide an alternative pathway for remethylation of homocysteine via BHMT in the methionine cycle (Fig. 4). When betaine treatment was used to normalize homocysteine levels of alcohol-fed mice there was a decrease in fatty liver and inflammation and suppression of the ER stress response, suggesting hyperhomocysteinemia is a trigger for endoplasmic reticulum stress [84].

Alterations in hepatic mitochondrial morphology and function are a hallmark of ALD. For example, alcohol-fed mice show an increased number of elongated mitochondria in liver and increased mitochondrial respiration [86]. These changes likely reflect an adaptive increase in the metabolism of alcohol. A separate proteomic study in livers of alcohol-fed rats detected decreases in several mitochondrial proteins involved in oxidative phosphorylation complexes. These decreases were prevented by co-feeding 
betaine with the ethanol, suggesting that preservation of mitochondrial function may be an additional mechanism by which betaine protects the liver from alcoholic injury [87]. Direct application of betaine to a hepatocyte cell line was reported to increase mitochondrial respiraton and ATP content although concentrations in the millimolar range were required. The mechanism was suggested to be indirect, possibly via intracellular signalling [88]. In other studies it has been suggested that betaine administration produces an increase in the rate of alcohol elimination through its indirect action to facilitate methylation of norepinephrine, converting it to epinephrine. The proposed mechanism is that epinephrine increases the metabolic rate to increase the $\mathrm{NAD}^{+} / \mathrm{NADH}$ ratio, providing more $\mathrm{NAD}^{+}$to catalyze destruction of ethanol by alcohol dehydrogenase [70].

Recent studies using an intestinal epithelial cell line showed that tight junctions were disrupted by ethanol and this effect was attenuated in the presence of betaine, suggesting that normal methylation activity helps to maintain tight junction integrity at least in an in vitro model [89]. It is possible to speculate, if alcohol and betaine have similar effects in vivo, that betaine offers additional protection by preventing pathogens in the gut from reaching the liver and contributing to hepatic inflammation and injury.

The progression of fatty liver to alcoholic hepatitis may be due to mechanisms triggered by metabolism of alcohol. These include disrupted methionine metabolism, induction of ER stress, increased susceptibility to TNF- $\alpha$, fibrosis and inflammation. Remarkably, as discussed above, betaine alleviates most of these problems, at least in experimental animals. It has even been suggested that the alcohol industry should add 
betaine to beer and wine so that drinkers could enjoy the effect of alcohol while reducing the risk of liver injury [70].

\section{Application to liver injury in humans}

The results of betaine administration to humans with liver injury have proved to be disappointing so far and underline the challenges in translating therapeutic options from animal studies to the human population. For example, in a 12-month study of 55 patients the administration of betaine did not improve hepatic steatosis or normalize liver enzymes in patients with NASH $[63,90]$. This should not be the last word since only 35 patients completed the study reducing the power to detect significant differences between the control and betaine-treated groups. In short, the study was underpowered and inconclusive which should not be interpreted as meaning there was no effect of betaine treatment on liver injury. It is also possible that additional factors may complicate studies of betaine action on liver injury in humans, and some will be considered here.

Before dealing with this issue it should be recognized that the liver processes $48 \%$ of dietary methionine to produce SAM [24]. As discussed earlier, BHMT and methionine synthase support the biosynthesis of methionine and SAM (Fig. 4). In most species, the majority of BHMT resides in the liver where its expression far exceeds that of other organs such as the kidney. In the liver it represents up to $2.0 \%$ of the total soluble protein [24]. Deletion of BHMT in mice had dramatic effects such as an 8-fold increase in plasma homocysteine, a 21 -fold increase in liver betaine content, and a $43 \%$ decrease in hepatic SAM content. The overall result was an estimated $75 \%$ reduction of methylation potential, and development of fatty liver within 5 weeks of age [22]. The importance of BHMT in hepatic betaine metabolism is further emphasized by its downregulation in 
response to hyperosmotic stress, at least in rat hepatoma and renal cells [91, 92]. This may reflect the normal high rate of betaine consumption through the BHMT pathway because consumption must be reduced in order to conserve enough betaine to fulfill its osmolyte role in cell volume regulation. If abnormal hepatic gene expression is a major factor underlying development and progression of fatty liver disease [93], then BHMT could occupy a central role for alleviating the problem by supplying one-carbon units from betaine for methylation of specific genes. If this is true then species differences in BHMT affinity for betaine might account in part for the apparent failure of betaine supplements to improve liver injury in humans. For example, a lower affinity of human BHMT for betaine might contribute to reduced effectiveness of betaine supplements for fatty liver injury. This might explain why very high doses of betaine ( $6 \mathrm{~g} /$ day or more) are required to reduce plasma homocysteine in humans. Values of $\mathrm{K}_{\mathrm{m}}$ and turnover numbers for BHMT taken from several publications are presented in Table 3. With betaine as substrate the $\mathrm{K}_{\mathrm{m}}$ values in rat and pig liver are somewhat variable but low, average $0.064 \mu \mathrm{M}$, suggesting apparent high affinity for betaine. Corresponding $\mathrm{K}_{\mathrm{m}}$ for human BHMT vary widely with some values similar to rat while others exceed $2.0 \mathrm{mM}$, a more than 30 -fold increase. In contrast the $\mathrm{k}_{\mathrm{cat}}$ for pig and human BHMT are comparable, based on the limited data. Some of the variability in $\mathrm{K}_{\mathrm{m}}$ for human BHMT has arisen even within the same research group and has been discussed but not explained [23]. Unfortunately direct comparisons of the kinetic constants of BHMT from animal and human liver determined under the same conditions in the same laboratory are not available. Note that the values reported in Table 3 are exclusively for BHMT and do not include the BHMT-2 isoform which cannot utilize betaine (see section 3). 
The betaine concentration in tissue water was estimated to be $0.4-4.1 \mathrm{mM}$ in rat liver $[1,31]$, assuming liver is $45 \%$ water by mass. Under these conditions, rat BHMT would be fully active providing the homocysteine level was adequate, which is another issue. Based on one study [94] homocysteine was estimated to be 4-8 $\mu \mathrm{M}$ in rat liver tissue water, below the range reported for the $\mathrm{K}_{\mathrm{m}}$ for homocysteine for BHMT (12-32 $\mu \mathrm{M})[95,96]$ in rat liver. Little is known about betaine and homocyteine concentrations in human liver. Given these variables, it is difficult to compare the level of BHMT enzyme activity in vivo in animal and human liver, and the utilization of betaine supplements by this activity in vivo. In summary, although there are some indications that the human enzyme has a lower apparent affinity (higher $\mathrm{K}_{\mathrm{m}}$ ) for betaine, the overall data remain equivocal (Table 3). The lack of improvement of human liver injury in response to betaine cannot be ascribed to different BHMT kinetics based on the evidence currently available.

Finally, in human studies it is not possible to verify directly that dietary betaine supplements lead to increased accumulation of betaine in the liver. Inadequate betaine uptake would lead to less than optimal responses to betaine treatment. Liver betaine uptake by BGT1, and other potential betaine transporters in liver such as SNAT and OCTN2 (see section 5), may be adversely affected by circulating metabolites which may be present in increased amounts due to liver disease. For example, a naturally occurring inhibitor of BGT1 is beta-alanine [50], a non-proteogenic amino acid which is produced endogenously in liver and also acquired through dietary intake. This and other, possibly unknown, factors also may impede betaine uptake via SNAT or OCTN2. Close attention to such issues may be necessary to optimize betaine treatment of human liver disease. 


\section{Conclusions}

In summary, betaine is effective in alleviating most of the disturbances in both NAFLD and ALD, at least in animal models. This may be due in part to the similarities in liver dysfunction (Table 2) and the effectiveness of betaine to reverse changes in fatty acid and lipid metabolism that, in turn, relieve ER stress and restore mitochondrial function. Some caution is needed in interpreting data from different laboratories because the different animal models are not always consistent in terms of species (rats or mice) and treatment regimens used to develop the models. In one study, for example, rats appear to be more resistant to some aspects of liver injury by ethanol compared to mice [97. Regarding the disappointing effects of betaine in humans, a better understanding of BHMT kinetics in human liver is needed in order to improve the effectiveness of betaine treatment. Also, the action of betaine supplements may be influenced by existing clinical conditions and diet [3] suggesting that additional factors and specific population groups may need to be considered. The potential significant benefits of betaine, due to its multiple effects, its low cost, and the safety of oral doses in the range $3-30 \mathrm{~g} / \mathrm{day}$, strongly suggest additional human trials should utilize larger groups for longer duration and should be rigorously controlled.

\section{Acknowledgements.}

We thank Dr Jeffrey Elmendorf, Indiana University School of Medicine, for careful review of the manuscript. Present address of Chris Day is George Washington University, Washington, DC. 


\section{References}

1. S. Slow, M. Lever, S.T. Chambers, P.M. George. Plasma dependent and independent accumulation of betaine in male and female rat tissues. Physiol. Res. 58 (2009) 403-410.

2. S.A. Craig. Betaine in human nutrition. Am. J. Clin. Nutr. 80 (2004) 539-549.

3. M. Lever, S. Slow. The clinical significance of betaine, an osmolyte with a key role in methyl group metabolism. Clin. Biochem. 43 (2010) 732-744.

4. S.H. Zeisel. Metabolic crosstalk between choline/1-carbon metabolism and energy homeostasis. Clin. Chem. Lab. Med. 51 (2013) 467-475.

5. V.R. Preedy (Ed.). Betaine: Chemistry, Analysis, Function and Effects, Royal Society of Chemistry, Cambridge UK, 2015.

6. J.H.F.G. Heijthuijsen, T.A. Hansen. Betaine fermentation and oxidation by marine desulfuromonas strains. Appl. Environ. Microbiol. 55 (1989) 965-969.

7. A.J. Watkins, E.G. Roussel, R.J. Parkes, H. Sass. Glycine betaine as a direct substrate for methanogens (Methanococcoides spp.). Appl. Environ. Microbiol. 80 (2014) 289-293.

8. P.H. Yancey. Organic osmolytes as compatible, metabolic and counteracting cytoprotectants in high osmolarity and other stresses. J. Exp. Biol. 208 (2005) 2819-2830.

9. D. Kultz, D. Chakravarty. Hyperosmolality in the form of elevated $\mathrm{NaCl}$ but not urea causes DNA damage in murine kidney cells. Proc. Nat. Acad. Sci. USA. 98 (2001) 1999-2004.

10. P. Bruździak, A. Panuszko, J. Stangret. Influence of Osmolytes on Protein and Water Structure: A Step To Understanding the Mechanism of Protein Stabilization. J. Phys. Chem. B. 117 (2013) 11502-11508.

11. D.W. Bolen, I.V. Baskakov. The osmophobic effect: natural selection of a thermodynamic force in protein folding. J. Mol. Biol. 310 (2001) 955-963.

12. L. Rigano, G. Dell'Acqua, R. Leporatti. Benefits of trimethylglycine (betaine) in personal-care formulations. Cosmetics Toiletries Mag. 115 (2000) 47-54.

13. A. Schiefner, J. Breed, L. Bösser, S. Kneip, J. Gade, G. Holtmann, K. Diederichs, W. Welte, E. Bremer. Cation- $\pi$ interactions as determinants for binding of the 
compatible solutes glycine betaine and proline betaine by the periplasmic ligandbinding protein ProX from Escherichia coli. J. Biol. Chem. 279 (204) 5588-5596.

14. M.B. Burg, J.D. Ferraris, N.I. Dmitrieva. Cellular response to hyperosmotic stresses. Physiol. Rev. 87 (2007) 1441-1474.

15. M.B. Burg, J.D. Ferraris. Intracellular Organic Osmolytes: Function and Regulation. J. Biol. Chem. 283 (2008) 7309-7313.

16. F. Beck, W. Neuhofer. Response of renal medullary cells to osmotic stress. Contrib. Nephrol. 148 (2005) 21-34.

17. W. Neuhofer, F.-X. Beck. Cell survival in the hostile environment of the renal medulla. Annu. Rev. Physiol. 67 (2005) 531-555.

18. Y. Zhou, S. Holmseth, R. Hua, A.C. Lehre, A.M. Olofsson, I. Poblete-Naredo, S.A. Kempson, N.C. Danbolt. The betaine-GABA transporter (BGT1, slc6a12) is predominantly expressed in the liver and at lower levels in the kidneys and at the brain surface. Am. J. Physiol. Renal Physiol. 302 (2012) F316-F328.

19. S.L. Anwar, U. Lehmann. DNA methylation, microRNAs, and their crosstalk as potential biomarkers in hepatocellular carcinoma. World J. Gastroenterol. 20 (2014) 7894-7913.

20. J.T. Fox, P.J. Stover. Folate mediated one carbon metabolism, in: L. Gerald (Ed.), Vitamins \& Hormones, Academic Press, Cambridge, 2008, vol. 79, pp. 1-44.

21. J.W. Locasale. Serine, glycine and one-carbon units: cancer metabolism in full circle. Nat. Rev. Cancer 13 (2013) 572-583.

22. Y.-W. Teng, M. Mehedint, T. Garrow, S. Zeisel. Deletion of betainehomocysteine S-methyltransferase in mice perturbs choline and 1-carbon metabolism, resulting in fatty liver and hepatocellular carcinomas. J. Biol. Chem. 286 (2011) 36258-36267.

23. N.S. Millian, T.A. Garrow. Human betaine-homocysteine methyltransferase is a zinc metalloenzyme. Arch. Biochem. Biophys. 356 (1998) 93-98.

24. M.A. Pajares, D. Pérez-Sala. Betaine homocysteine S-methyltransferase: just a regulator of homocysteine metabolism? Cell. Mol. Life Sci. 63 (2006) 2792-2803.

25. J. Mládková, J. Hladílková, C.E. Diamond, K. Tryon, K. Yamada, T.A. Garrow, P. Jungwirth, M. Koutmos, J. Jiráček. Specific potassium ion interactions 
facilitate homocysteine binding to betaine-homocysteine S-methyltransferase. Proteins 82 (2014) 2552-2564.

26. F. Li, Q. Feng, C. Lee, S. Wang, L.L. Pelleymounter, I. Moon, B.W. Eckloff, E.D. Wieben, D.J. Schaid, V. Yee, R.M. Weinshilboum. Human betaine-homocysteine methyltransferase (BHMT) and BHMT2: Common gene sequence variation and functional characterization. Mol. Gen. Metab. 94 (2008) 326-335.

27. S.S. Szegedi, C.C. Castro, M. Koutmos, T.A. Garrow. Betaine-homocysteine Smethyltransferase-2 is an S-methylmethionine-homocysteine methyltransferase. J. Biol. Chem. 283 (2008) 8939-8945.

28. R.F. Bertolo, L.E. McBreairty. The nutritional burden of methylation reactions. Curr. Opin. Clin. Nutr. Metab. Care 16 (2013) 102-108.

29. X. Xua, J.G. Chen. One-carbon metabolism and breast cancer: an epidemiological perspective. J. Genet. Genomics 36 (2009) 203-214.

30. P.C. Nicholas, D. Kim, F.T. Crews, J.M. Macdonald. ${ }^{1}$ H NMR-based metabolomic analysis of liver, serum, and brain following ethanol administration in rats. Chem. Res. Toxicol. 21 (2008) 408-420.

31. S.A. Kempson, K. Vovor-Dassu, C. Day. Betaine transport in kidney and liver: use of betaine in liver injury. Cell. Physiol. Biochem. 32 (2013) 32-40.

32. G.S. Sharma, T. Kumar, T.A. Dar, and L.R. Singh. Protein N-homocysteinylation: From cellular toxicity to neurodegeneration. Biochim. Biophys. Acta 1850 (2015) 2239-2245.

33. European Food Safety Authority. Scientific opinion on the safety and efficacy of betaine (betaine anhydrous and betaine hydrochloride) as a feed additive for all animal species. Eur. Food Safety Auth. J. 11 (2013) 3210.

34. M. Eklund, E. Bauer, J. Wamatu, R. Mosenthin. Potential nutritional and physiological functions of betaine in livestock. Nutrition Res. Rev. 18 (2005) 3148.

35. U. Schwab, A. Törrönen, L. Toppinen, G. Alfthan, M. Saarinen, A. Aro, M. Uusitupa. Betaine supplementation decreases plasma homocysteine concentrations but does not affect body weight, body composition, or resting energy expenditure in human subjects. Am. J. Clin. Nutr. 76 (2002) 961-967. 
36. M.K.I. Anas, M.B. Lee, C. Zhou, M.-A. Hammer, S. Slow, J. Karmouch, X.J. Liu, S. Bröer, M. Lever, J.M. Baltz. SIT1 is a betaine/proline transporter that is activated in mouse eggs after fertilization and functions until the 2-cell stage. Development 135 (2008) 4123-4130.

37. M.B. Lee, M. Kooistra, B. Zhang, S. Slow, A.L. Fortier, T.A. Garrow, M. Lever, J.M. Trasler, J.M. Baltz. Betaine homocysteine mhethyltransferase is active in the mouse blastocyst and promotes inner cell mass development. J. Biol. Chem. 287 (2012) 33094-33103.

38. S.T. Lamitina, K. Strange. Transcriptional targets of DAF-16 insulin signaling pathway protect C. elegans from extreme hypertonic stress. Am. J. Physiol. Cell Physiol. 288 (2005) C467-474.

39. A. Peden, P. Mac, Y.-J. Fei, C. Castro, G. Jiang, K. Murfitt, E. Miska, J. Griffin, V. Ganapathy, E. Jorgensen. Betaine acts on a ligand-gated ion channel in the nervous system of the nematode Nat. Neurosci. 16 (2013) 1794-1801.

40. D. Cleland, P. Krader, C. McCree, J. Tang, D. Emerson. Glyceine betaine as a cryoprotectant for prokaryotes. J. Microbiol. Methods 58 (2004) 31-38.

41. E. Virtanen, M. Junnila, A. Soivio. Effects of food containing betaine/amino acid additive on the osmotic adaptation of young Atlantic salmon, Salmo salar. Aquacultur. 83 (1989) 109-122.

42. W.C. Clarke, E. Virtanen, J. Blackburn, D.A. Higgs. Effects of a dietary betaine/amino acid additive on growth and seawater adaptation in yearling chinook salmon. Aquaculture 121 (1994) 137-145.

43. W.A. Rees, T.D. Yager, J. Korte, P.H. von Hippel. Betaine can eliminate the base pair composition dependence of DNA melting. Biochemistry 32 (1993)137-144.

44. W. Henke, K. Herdel, K. Jung, D. Schnorr, S.A. Loening. Betaine improves the PCR amplification of GC-rich DNA sequences. Nucleic Acids Res. 25 (1997) 3957-3958.

45. P.N. Hengen. Optimizing multiplex and LA-PCR with betaine. Trends Biochem. Sci. 22 (1997) 225-226. 
46. S. Pummer, W. Dantzler, Y. Lien, G. Moeckel, K. Volker, S. Silbernagl. Reabsorption of betaine in Henle's loops of rat kidney in vivo. Am. J. Physiol. Renal Physiol. 278 (2000) F434-F439.

47. M. Cano, M.L. Calonge, A.A. Ilundáin. $\mathrm{Na}^{+}$-dependent and $\mathrm{Na}^{+}$-independent betaine transport across the apical membrane of rat renal epithelium. Biochim. Biophys. Acta 1848 (2015) 2172-2179.

48. M.B. Burg, H.M. Kwon, D. Kultz. Osmotic regulation of gene expression. FASEB J. 10 (1996) 1598-1606.

49. M.S. Kwon, S.W. Lim, H.M. Kwon. Hypertonic stress in the kidney: a necessary evil. Physiology 24 (2009) 186-191.

50. S.A. Kempson, Y. Zhou, N.C. Danbolt. The betaine/GABA transporter and betaine: roles in brain, kidney and liver. Front. Physiol. 5 (2014) doi 10.3389/fphys.2014.00159.

51. S. Kempson, B. Anderson, M. Levi, J. Blaine. Nitric oxide activates plasma membrane insertion of the betaine/GABA transporter in renal epithelial cells. Res. Cell Biol. 2 (2014) 1-7.

52. C. Perego, A. Bulbarelli, R. Longhi, M. Caimi, A. Villa, M.J. Caplan, G. Pietrini. Sorting of two polytopic proteins, the GABA and betaine transporters, in polarized epithelial cells. J. Biol. Chem. 272 (1997) 6584-6592.

53. C. Perego, C. Vanoni, A. Villa, R. Longhi, S.M. Kaech, E. Frohli, A. Hajnal, S.K. Kim, G. Pietrini. PDZ-mediated interactions retain the epithelial GABA transporter on the basolateral surface of polarized epithelial cells. EMBO J. 18 (1999) 2384-2393.

54. C.R. Day, S.S. Gordon, C.L. Vaughn, S.A. Kempson. A single amino acid substitution in the renal betaine/GABA transporter prevents trafficking to the plasma membrane. Physiol. J. (2013) Article ID 598321 http://dx.doi.org/10.1155/2013/598321

55. E.S. Schweikhard, S.A. Kempson, C. Ziegler, B.C. Burckhardt. Mutation of a single threonine in the cytoplasmic $\mathrm{NH}_{2}$ terminus disrupts trafficking of renal betaine-GABA transporter 1 during hypertonic stress. Am. J. Physiol. Renal Physiol. 307 (2014) F107-F115. 
56. E.S. Schweikhard, B.C. Burckhardt, F. Joos, C. Fenollar-Ferrer, L.R. Forrest, S.A. Kempson, C. Ziegler. Role of N-glycosylation in renal betaine transport. Biochem. J. 470 (2015) 169-179.

57. C. Perez, C. Koshy, O. Yildiz, C. Ziegler. Alternating-access mechanism in conformationally asymmetric trimers of the betaine transporter BetP. Nature 490 (2012) 126-130.

58. K.A. Clow, J.R. Treberg, M.E. Brosnan, J.T. Brosnan. Elevated tissue betaine contents in developing rats are due to dietary betaine, not to synthesis. J. Nutr. 138 (2008) 1641-1646.

59. J. Chen, X. Zhou, W. Wu, X. Wang, Y. Wang. FTO-dependent function of N6methyladenosine is involved in the hepatoprotective effects of betaine on adolescent mice. J. Physiol. Biochem. 71 (2015) 405-413.

60. M. Basaranoglu, G. Basaranoglu, H. Sentürk. From fatty liver to fibrosis: A tale of "second hit". World J. Gastroenterol. 19 (2013) 1158-1165.

61. M. Duvnjak, I. Lerotić, N. Baršić, V. Tomašić, L.V. Jukić, V. Velagić. Pathogenesis and management issues for non-alcoholic fatty liver disease. World J. Gastroenterol. 13 (2007) 4539-4550.

62. R. Deminice, R.P. da Silva, S.G. Lamarre, K.B. Kelly, R.L. Jacobs, M.E. Brosnan, J.T. Brosnan. Betaine supplementation prevents fatty liver induced by a high-fat diet: effects on one-carbon metabolism. Amino Acids 47 (2015) 839-846.

63. S. Mukherjee. Betaine and nonalcoholic steatohepatitis: back to the future? World J. Gastroenterol 17 (2011) 3663-3664.

64. M. Gulsen, Z. Yesilova, S. Bagci, A. Uygun, A. Ozcan, C.N. Ercin, A. Erdil, S.Y. Sanisoglu, E. Cakir, Y. Ates, M.K. Erbil, N. Karaeren, K. Dagalp. Elevated plasma homocysteine concentrations as a predictor of steatohepatitis in patients with non-alcoholic fatty liver disease. J. Gastroenterol. Hepatol. 20 (2005)14481455.

65. S. de Carvalho, M. Muniz, M. Siqueira, E. Siqueira, A. Gomes, K. Silva, L. Bezerra, V. D'Almeida, C. de Oliveira, L. Pereira. Plasmatic higher levels of homocysteine in non-alcoholic fatty liver disease (NAFLD). Nutr. J. 12 (2013) doi: 10.1186/1475-2891-12-37 
66. D. Pessayre. Role of mitochondria in non-alcoholic fatty liver disease. J Gastroenterol. Hepatol. 22 (2007) S20-S27.

67. W. Xiao, M. Ren, C. Zhang, S. Li, W. An. Amelioration of nonalcoholic fatty liver disease by hepatic stimulator substance via preservation of carnitine palmitoyl transferase-1 activity. Am. J. Physiol. Cell Physiol. 309 (2015) C215C227.

68. H. Tilg, A.M. Diehl. Cytokines in Alcoholic and Nonalcoholic Steatohepatitis. New Engl. J. Med. 343 (2000) 1467-1476.

69. D. Schuppanand, N.H. Afdhal. Liver cirrhosis. Lancet 371 (2008) 38-851.

70. S.W. French. How to prevent alcoholic liver disease. Exp. Mol. Pathol. 98 (2015) 304-307.

71. M.R. Olthof, T. van Vliet, E. Boelsma, P. Verhoef. Low dose betaine supplementation leads to immediate and long term lowering of plasma homocysteine in healthy men and women. J. Nutr. 133 (203) 4135-4138.

72. Z. Song, I. Deaciuc, Z. Zhou, M. Song, T. Chen, D. Hill, C.J. McClain. Involvement of AMP-activated protein kinase in beneficial effects of betaine on high-sucrose diet-induced hepatic steatosis. Am. J. Physiol. Gastrointest. Liver Physiol. 293 (2007) G894-G902.

73. E. Kathirvel, K. Morgan, G. Nandgiri, B.C. Sandoval, M.A. Caudill, T. Bottiglieri, S.W. French, T.R. Morgan. Betaine improves nonalcoholic fatty liver and associated hepatic insulin resistance: a potential mechanism for hepatoprotection by betaine. Am. J. Physiol. Gastrointest. Liver Physiol. 299 (2010) G1068-G1077.

74. Z. Wang, T. Yao, M. Pini, Z. Zhou, G. Fantuzzi, Z. Song. Betaine improved adipose tissue function in mice fed a high-fat diet: a mechanism for hepatoprotective effect of betaine in nonalcoholic fatty liver disease. Am. J. Physiol. Gastrointest. Liver Physiol. 298 (2010) G634-G642.

75. L. Xu, D. Huang, Q. Hu, J. Wu, Y. Wang, J. Feng. Betaine alleviates hepatic lipid accumulation via enhancing hepatic lipid export and fatty acid oxidation in rats fed with a high-fat diet. Br. J. Nutr. 113 (2015) 1835-1843. 
76. M. You, D.W. Crabb. Recent advances in alcoholic liver disease II. Minireview: molecular mechanisms of alcoholic fatty liver. Am. J. Physiol. Gastrointest. Liver Physiol. 287 (2004) G1-G6.

77. D.W. Crabb, S. Liangpunsakul. Alcohol and lipid metabolism. J. Gastroenterol. Hepatol. 21 (2006) S56-S60.

78. J. García-Villafranca, A. Guillén, J. Castro. Ethanol consumption impairs regulation of fatty acid metabolism by decreasing the activity of AMP-activated protein kinase in rat liver. Biochimie 90 (2008) 460-466.

79. M. You, M. Matsumoto, C.M. Pacold, W.K. Cho, D.W. Crabb. The role of AMPactivated protein kinase in the action of ethanol in the liver. Gastroenterol. 127 (2004) 1798-1808.

80. K. Tomita, G. Tamiya, S. Ando, N. Kitamura, H. Koizumi, S. Kato, Y. Horie, T. Kaneko, T. Azuma, H. Nagata, H. Ishii, T. Hibi. AICAR, an AMPK activator, has protective effects on alcohol-induced fatty liver in rats. Alcohol Clin. Exp. Res. 29 (2005) 240S-245S.

81. A.J. Barak, H.C. Beckenhauer, S. Badakhsh, D.J. Tuma. The effect of betaine in reversing alcoholic steatosis. Alcohol Clin. Exp. Res. 21 (1997) 1100-1102.

82. C. Ji, N. Kaplowitz. Betaine decreases hyperhomocysteinemia, endoplasmic reticulum stress, and liver injury in alcohol-fed mice. Gastroenterol. 124:14881499 (2003).

83. K.K. Kharbanda, M.E. Mailliard, C.R. Baldwin, H.C. Beckenhauer, M.F. Sorrell, D.J. Tuma. Betaine attenuates alcoholic steatosis by restoring phosphatidylcholine generation via the phosphatidylethanolamine methyltransferase pathway. J Hepatol. 46 (2007) 314-321.

84. N. Kaplowitz, C. Ji. Unfolding new mechanisms of alcoholic liver disease in the endoplasmic reticulum. J. Gastroenterol. Hepatol. 21 (2006) S7-S9.

85. K.K. Kharbanda. Alcoholic liver disease and methionine metabolism. Semin. Liver Dis. 29 (2009) 155-165.

86. D. Han, M.D. Ybanez, H.S. Johnson, J.N. McDonald, L. Mesropyan, H. Sancheti, G. Martin, A. Martin, A.M. Lim, L. Dara, E. Cadenas, H. Tsukamoto, N. Kaplowitz. Dynamic adaptation of liver mitochondria to chronic alcohol feeding 
in mice: biogenesis, remodeling, and functional alterations. J. Biol. Chem. 287 (2012) 42165-42179.

87. K.K. Kharbanda, S.L. Todero, A.L. King, N.A. Osna, B.L. McVicker, D.J. Tuma, J.L. Wisecarver, S.M. Bailey. Betaine treatment attenuates chronic ethanolinduced hepatic steatosis and alterations to the mitochondrial respiratory chain proteome. Int. J. Hepatol. (2012) Article ID 962183. http://dx.doi.org/10.1155/2012/962183.

88. I. Lee. Betaine is a positive regulator of mitochondrial respiration. Biochem. Biophys. Res. Commun. 456 (2015) 621-625.

89. P.G. Thomes, N.A. Osna, S.M. Bligh, D.J. Tuma, K.K. Kharbanda. Role of defective methylation reactions in ethanol-induced dysregulation of intestinal barrier integrity. Biochem. Pharmacol. 96 (2015) 30-38.

90. M. Abdelmalek, S. Sanderson, P. Angulo, C. Soldevila-Pico, C. Liu, J. Peter, J. Keach, M. Cave, T. Chen, C. McClain, K. Lindor. Betaine for nonalcoholic fatty liver disease: results of a randomized placebo-controlled trial. Hepatol. 50 (2009) 1818-1826.

91. R.W. Grunewald, A. Eckstein. Osmotic regulation of the betaine metabolism in immortalized renal cells. Kidney Int. 48 (1995) 1714-1720.

92. C. Schafer, L. Hoffmann, K. Heldt, M.R. Lornejad-Schafer, G. Brauers, T. Gehrmann, T.A. Garrow, D. Haussinger, E. Mayatepek, B.C. Schwahn, F. Schliess. Osmotic regulation of betaine homocysteine-S-methyltransferase expression in H4IIE rat hepatoma cells. Am. J. Physiol. Gastrointest. Liver Physiol. 292 (2007) G1089-1098.

93. L.-j. Wang, H.-w. Zhang, J.-y. Zhou, Y. Liu, Y. Yang, X.-1. Chen, C.-h. Zhu, R.d. Zheng, W.-h. Ling, H.-l. Zhu. Betaine attenuates hepatic steatosis by reducing methylation of the MTTP promoter and elevating genomic methylation in mice fed a high-fat diet. J. Nutr. Biochem. 25 (2014) 329-336.

94. P.M. Ueland, S. Helland, O.J. Broch, J.S. Schanche. Homocysteine in tissues of the mouse and rat. J. Biol. Chem. 259 (1984) 2360-2364. 
95. J.D. Finkelstein, B.J. Harris, W.E. Kyle. Methionine metabolism in mammals: kinetic study of betaine-homocysteine methyltransferase. Arch. Biochem. Biophys. 153 (1972) 320-324.

96. T.A. Garrow. Purification, kinetic properties, and cDNA cloning of mammalian betaine-homocysteine methyltransferase. J. Biol. Chem. 271 (1996) 22831-22838.

97. M. Shinohara, C. Ji, N. Kaplowitz. Differences in betaine-homocysteine methyltransferase expression, endoplasmic reticulum stress response, and liver injury between alcohol-fed mice and rats. Hepatol. 51 (2010) 796-805.

98. M.J. O'Neil (Ed.). The Merck Index - An Encyclopedia of Chemicals, Drugs, and Biologicals, Merck and Co. Inc., Whitehouse Station, NJ, 2006.

99. K.-H. Lee, M. Cava, P. Amiri, T. Ottoboni, R.N. Lindquist. Betaine:Homocysteine methyltransferase from rat liver: Purification and inhibition by a boronic acid substrate analog. Arch. Biochem. Biophys. 292 (1992) 77-86.

100. W.E. Skiba, M.S. Wells, J.H. Mangum, W.M. Awad. Betaine-homocysteine Smethyltransferase (human). Methods Enzymol. 143 (1987) 384-388.

101. W.E. Skiba, M.P. Taylor, M.S. Wells, J.H. Mangum, W.M. Awad. Human hepatic methionine biosynthesis. Purification and characterization of betaine:homocysteine S-methyltransferase. J. Biol. Chem. 257 (1982) 1494414948.

102. C. Castro, A.A. Gratson, J.C. Evans, J. Jiracek, M. Collinsová, M.L. Ludwig, T.A. Garrow. Dissecting the catalytic mechanism of betaine-homocysteine Smethyltransferase by use of intrinsic tryptophan fluorescence and site-directed mutagenesis. Biochemistry 43 (2004) 5341-5351. 


\begin{tabular}{|l|l|}
\hline CAS number & $107-43-7$ \\
\hline Molecular formula & $\mathrm{C}_{5} \mathrm{H}_{11} \mathrm{NO}_{2}$ \\
\hline Molecular wt & 117.1 \\
\hline Color & White hygroscopic crystals \\
\hline Taste & Sweet \\
\hline Solubility & Water: 160. Methanol: 57. Ethanol 8.7. \\
\hline pKa & Aqueous solution is clear and colorless. \\
\hline Melting point & 1.83 at $0{ }^{\circ} \mathrm{C}$ \\
\hline LD $D_{50}$ & $293{ }^{\circ} \mathrm{C}$ (decomposes) \\
\hline
\end{tabular}

Table 1. Physical and chemical properties of betaine.

Date from reference [98]. 


\begin{tabular}{|l|}
\hline \multicolumn{1}{|c|}{ Liver disturbances common to both NAFLD } \\
\hline Insulin resistance \\
\hline Dysregulation of genes involved in lipid metabolism \\
\hline Steatosis \\
\hline Mitochondrial dysfunction \\
\hline ER stress \\
\hline Decreased AMPK activity \\
\hline Proinflammatory cytokines (e.g.TNF- $\alpha$ ) released \\
\hline Hyperhomocysteinemia \\
\hline
\end{tabular}

Table 2. Overlapping characteristics of liver dysfunction in non-alcoholic fatty liver disease (NAFLD) and alcoholic liver disease (ALD).

Although the disease courses are quite divergent, the functional and histological disturbances are similar in NAFLD and ALD models, based on reports from many different laboratories. In rodent models NAFLD was induced by a high fat (or high sucrose) diet, and ALD was induced by chronic ethanol feeding. Almost all the disturbances, especially in ALD, were attenuated or reversed by dietary betaine supplements. See text, section 7, for details. 


\begin{tabular}{|c|c|c|c|}
\hline \multicolumn{2}{|c|}{ Rat or pig liver } & \multicolumn{2}{c|}{ Human liver } \\
\hline $\begin{array}{c}\mathrm{K}_{\mathrm{m}} \text { for betaine } \\
(\mathrm{mM})\end{array}$ & $\begin{array}{c}\mathrm{k}_{\text {cat }} \text { for betaine } \\
\left(\mathrm{h}^{-1}\right)\end{array}$ & $\begin{array}{c}\mathrm{K}_{\mathrm{m}} \text { for betaine } \\
(\mathrm{mM})\end{array}$ & $\begin{array}{c}\mathrm{k}_{\text {cat }} \text { for betaine } \\
\left(\mathrm{h}^{-1}\right)\end{array}$ \\
\hline 0.023 (pig) $[96]$ & 90 & $0.023[26]$ & \\
\hline 0.048 (rat) [95] & & $0.075[25]$ & 107 \\
\hline 0.12 (rat) [99] & & $0.10[100]$ & \\
& & $0.12[101]$ & \\
\hline & & $1.7[102]$ & \\
\hline & & $2.2[27]$ & \\
\hline & & $2.47[23]$ & \\
\hline
\end{tabular}

Table 3. Comparison of Michaelis constant $\left(K_{m}\right)$ and turnover number $\left(k_{c a t}\right)$ for betaine homocysteine methyltransferase (BHMT) from animal and human liver. 
The kinetic constants determined with betaine as the substrate are taken from data published by several different laboratories. References in parentheses. Data for BHMT2 are not included because this isoform cannot utilize betaine (see text, section 3). 
Figure legends
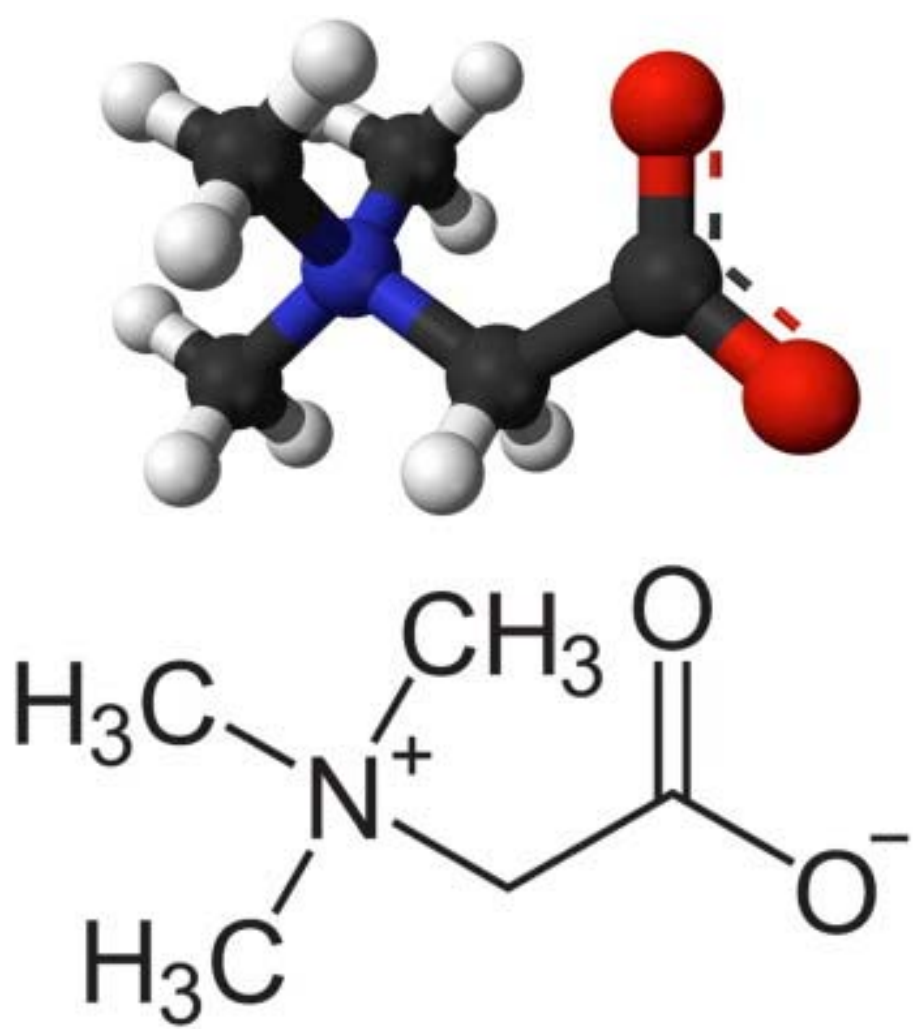

Fig. 1. Betaine structure

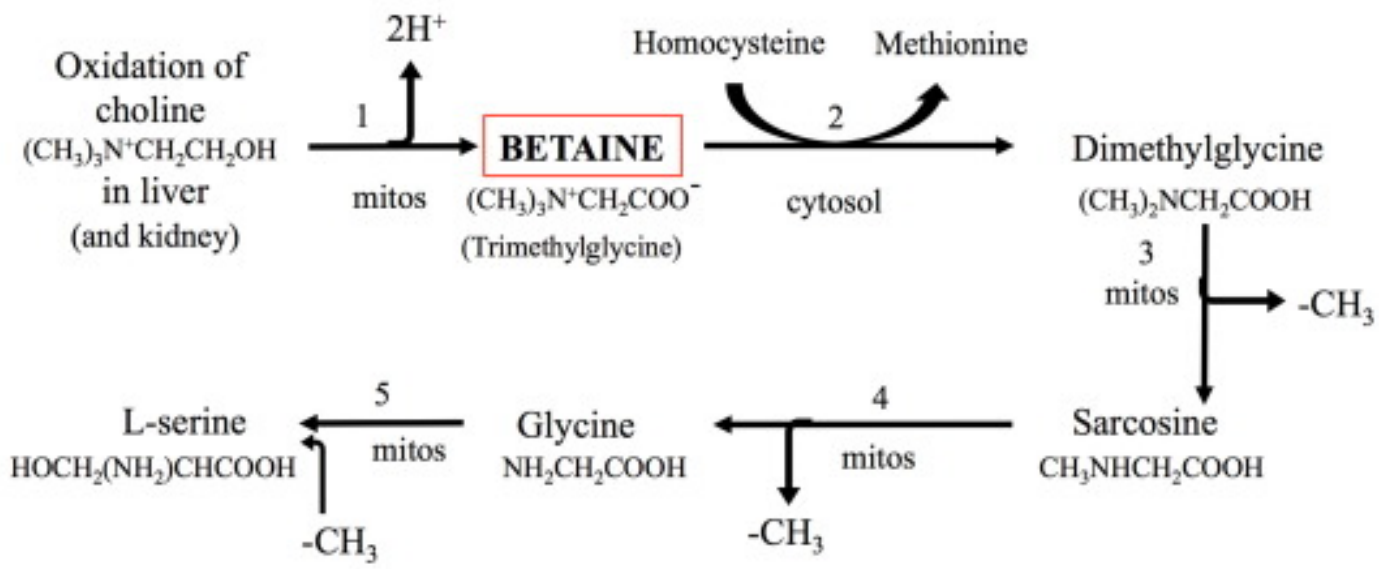

Fig. 2. Pathway of betaine metabolism in liver. Choline from dietary intake is absorbed in the intestine and transported to the liver and kidneys. In the liver it is either 
incorporated into phosphatidylcholine or converted into betaine. In the latter case, the first step (1) is oxidation in mitochondria via two reactions catalyzed by choline dehydrogenase (EC 1.1.99.1) followed by betaine aldehyde dehydrogenase (EC1.2.1.8). Betaine degradation continues in the cytosol (2) and requires betaine-homocysteine methyltransferase (BHMT, EC2.1.1.5). Step 3 is catalyzed by dimethylglycine dehydrogenase ((EC 1.5.99.2) in mitochondria. Step 4 also in mitochondria requires sarcosine dehydrogenase (EC1.5.99.1). The final step (5) mediated by serine hydroxymethyltransferase (EC 2.1.2.1.) occurs in both mitochondria and cytosol. Overall, use of betaine for methylation of homocysteine by BHMT (step 2) recovers a methyl group to the methionine cycle and the remaining methyl groups enter the one-carbon pool. 


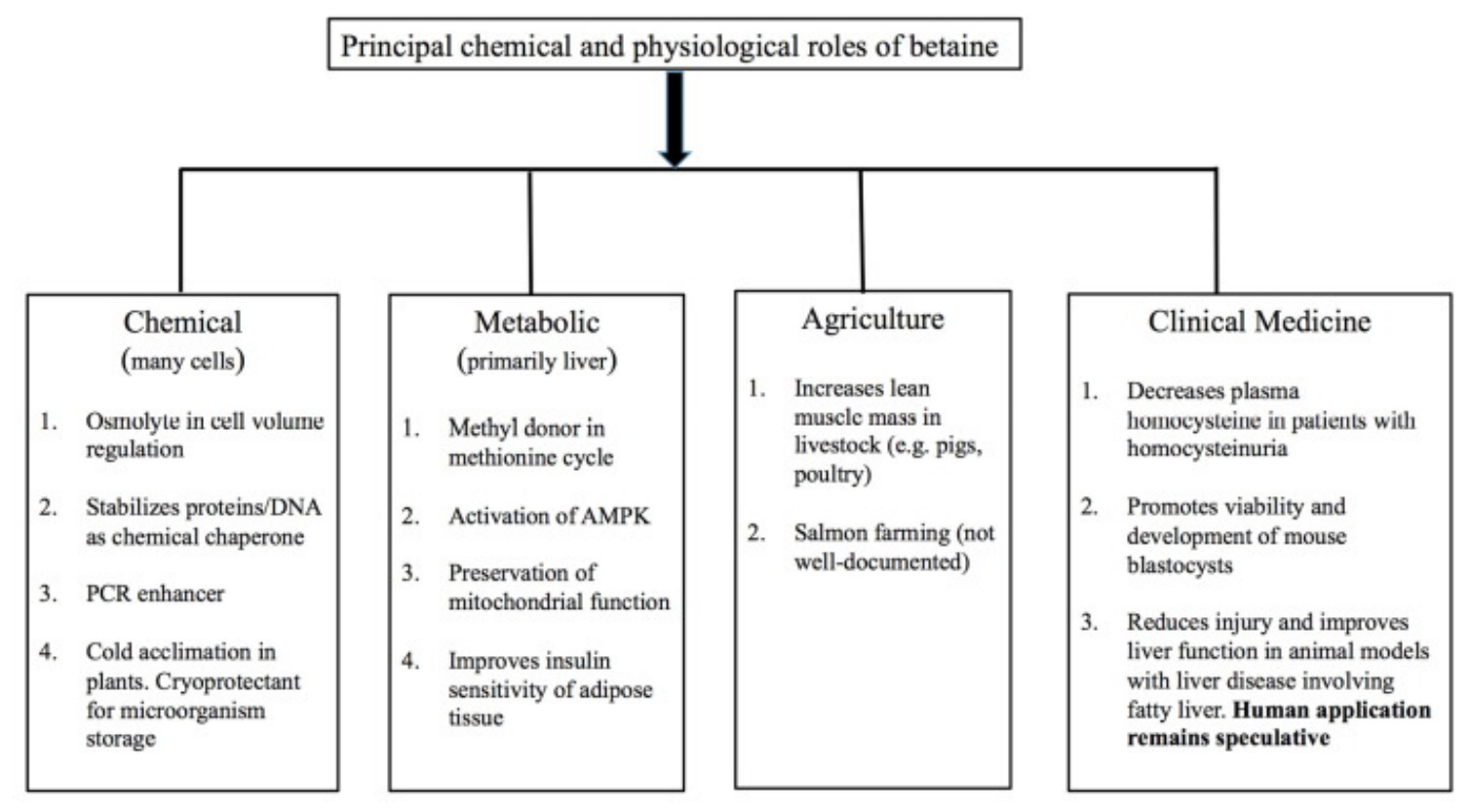

Fig. 3. Diverse roles of betaine. See text, sections 3-4, for details.

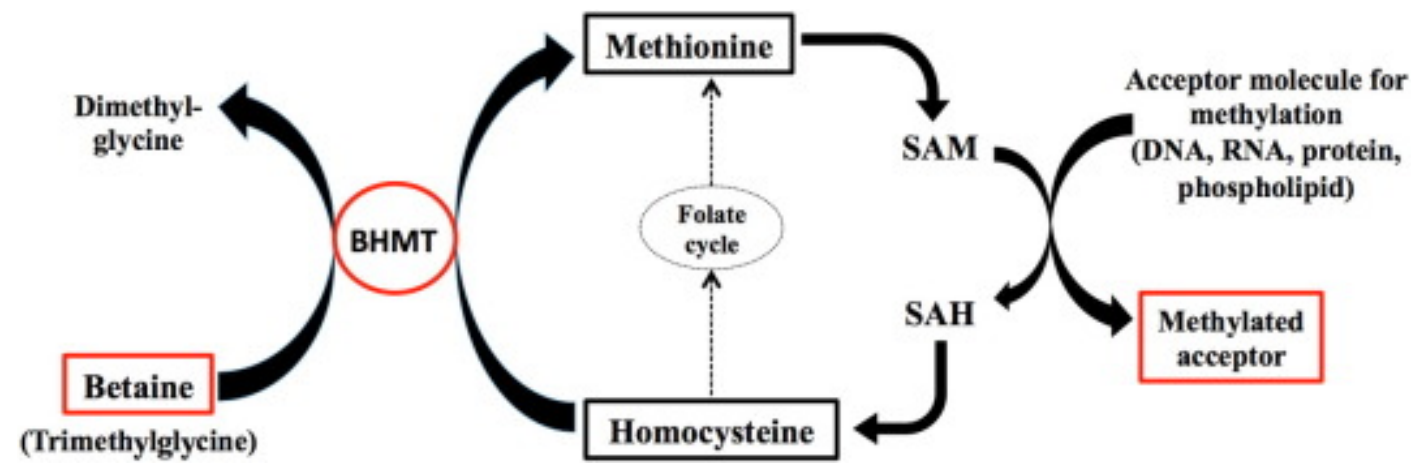

Fig. 4. Methionine cycle in liver. Conversion of homocysteine to methionine also occurs via the folate cycle (not shown in detail) which uses 5-methyltetrahydrofolate as a methyl donor in a reaction mediated by methionine synthase (EC 2.1.1.13) $(2,21)$. See text, section 3, for further information. BHMT, betaine-homocysteine methyltransferase. DMG, dimethylglycine. SAM, S-adenosylmethionine. SAH, S-adenosylhomocysteine. 